powerful method of identifying clay minerals, and due acknowledgments are made to the Bragg school, Koss and Kerr, Pauling, Hendricks and Nagelschmidt for their pioneer work. The value of auxiliary techniques is emphasized, and well-balanced accounts are included of chemical, optical and thermal studies. Mechanical analysis, aided by the centrifuge, is a necessary prelude to the application of X-ray diffraction, chemical and thermal techniques to the quantitative study of clay, soil and ocean deposits. Chapters of a more advanced nature describe crystal structure studies of montmorillonite, halloysite, fireclay and chamosite, and $\mathrm{X}$-ray studies of interstratified and disordered layer lattice minerals. A knowledge of the silicon-aluminium oxygen layer framework and where the layers of water molecules are located can often be extended to a more detailed account of where additional elements-potassium, sodium, magnesium, iron, etc.-are also situated. Thus are explained many of the known properties of clay minerals. It is not too much to expect that more exact crystal structure analyses will lead to the prediction of unsuspected properties, some of which may be useful to science, art and industry.

F. A. Bannister

\section{CHEMISTRY OF LIGNIN AND WOOD}

The Chemistry of Lignin

By Friedrich Emil Brauns. Pp. xv +808 . (New York : Academic Press, Inc., 1952.) 14.50 dollars.

\section{Chemistry of Wood}

By Erik Hägglund. Pp. $x+631$. (New York: Academic Press, Inc., 1951.) 13.50 dollars.

1 HESE two books have much in common. Each has as author a prominent member of a pulp and paper research organization-Brauns being of the Institute of Paper Chemistry, Wisconsin, and Hägglund of the Swedish Forest Products Research Laboratory-and both authors are prominent authorities on the subject-matter covered. Both books, too, are essentially documentary in character and contain some 2,200 and 1,400 references to the original literature, respectively. Peculiarly enough, the same (and indeed, only) adverse comment applies to both - namely, that they are, if anything, too documentary and perhaps insufficiently critical of the work recorded, including the author's own.

The chemistry of lignin is in effect largely the chemistry of wood, so that overlaps are to be expected and indeed make comparison a matter of some interest. For example, sections on the structure of lignin and its formation and role in the plant are common to the two volumes. Both books adopt an essentially chemical approach to their subject, although the wider industrial implications are duly recognized, even if they are not fully discussed as such. Thus, Brauns emphasizes how the academic chemical approach to the problem of lignin structure via its chemical reactions should lead to the solution of that major problem of all pulping industries, namely, the economic utilization of lignin instead of its disposal as a highly objectionable effluent. Similarly, Hägglund's book has chapters on the chemistry of the acid and alkaline pulping processes used for the manufacture of wood pulp for paper and rayon manufacture.
As might be expected, Brauns's book is the more restricted in scope although, in consequence, each section is correspondingly more detailed. Aspects covered are the analysis of lignin, its isolation, and its physical, biological and chemical properties and reactions, together with its role and formation in the plant. A long chapter deals, of course, with the various theories of the structure of lignin. Unfortunately, it leads to the conclusion that it is premature to suggest a constitutional formula for lignin, although there is strong evidence that lignin is a high polymer comprising at least 75 per cent of phenylpropane 'building stones'. The structure of these 'building stones' and the mode of their combination are, however, still unknown, and indeed Brauns regards our knowledge of the structure of lignin as comparable with that of cellulose more than a hundred years ago.

Hägglund's book is a revised edition of "Holzchemie", first published in 1928 (second German edition, 1939), now, significantly enough, appearing in English although printed in Sweden and published in the United States. The ground covered includes wood tissue and formation, the physical and chemical properties of wood and its various constituents, the chemistry of wood saccharification (Rheinau process), and the sulphite and alkaline pulping methods and associated bleaching processes. The carbonization, caustic fusion, storage and natural decomposition of wood are also dealt with.

It is noteworthy, and a tribute to both the authors and publishers, that although the books are printed in Vienna and Sweden, respectively, the standard of production bears very favourable comparison with the best of similar books published in Great Britain. These two books, though similar, can be highly recommended as full, authoritative and up-to-date accounts of our present knowledge of wood and its constituents. On the whole, they are complementary to, rather than substitutes for, one another; and studied together they should meet every need of all workers on the subjects of wood and lignin.

JuLIUS GRANT

\section{ABSTRACTING FOR PHYSICISTS AND ELECTRICAL ENGINEERS}

\section{Physics Abstracts}

Section A of Science Abstracts. Vol. 54, 1951. Pp. vii +1460.

\section{Electrical Engineering Abstracts}

Section B of Science Abstracts. Vol. 54, 1951. Pp. $\mathrm{xii}+737$.

(London : Institution of Electrical Engineers, 1951.) $£ 210$ s. each section; $£ 410 s$. both sections.

IN 1948 the Royal Society Scientific Information Conference in London discussed among other topics the question of improvement of abstracting services; in 1949 Unesco convened a conference in Paris to discuss the co-ordination of the various science abstract journals, and in 1950 the results of an extensive and detailed study, undertaken by the American Institute of Physics with the financial assistance of the U.S. Office of Naval Research, of the opinions of physicists on Physics Abstracts (Science Abstracts A) were published (Amer. J. Phys., 18, 417; October 1950. See also Physics Today, 4, 18 ; August 1951). The principal object of the latter inquiry was to ascertain for what purposes physicists use abstracts, their opinion of the abstracting services 